\section{Metacognitive competence as a goal for medical training}

\author{
Alessandro Antonietti, \\ Valentina Rita Andolfi, Barbara Colombo \\ Department of Psychology, Catholic \\ University of the Sacred Heart, Milan, \\ Italy
}

\section{Abstract}

Professionals who are faced with emergency situations daily during their work can rely on three different ways of thinking. They can base their judgments and decisions on intuition. Alternatively they can apply heuristic strategies, which offer simple procedures to simplify situations and find satisfactory solutions. Finally, they can reflect analytically. The optimal approach would be a flexible use of these three systems, since it enables doctors to activate the system that is more relevant to the given situation and eventually to pass to another system when they realize that the previous one is inadequate. Metacognitive competence is required in order to identify the mental system that is more relevant to a specific case. This competence consists in the ability to self-regulate cognitive processes in order to match the specific needs of the moment. To do so, individuals have to pay attention to their cognitive processes and understand how they can be trusted and what is the best way to handle them. Operatively, metacognitive competence should be developed by leading professionals to identify the mode of thinking - intuitive, heuristic or analytical - that is best suited to make the choices required by the clinical cases that they are facing. Suggestions concerning the way physicians working in emergency department can be trained to enhance their metacognitive skills are reported.

\section{Introduction}

Traditional theories of decision making suggest that optimal choices derive from a careful examination of the available data and rely on a thoughtful assessment of the options to be chosen. Unfortunately, emergency medicine does not always provide the conditions to proceed this way. When decisions have to be made quickly and without all the necessary data, apparently the alternative is to rely on similarities between a specific clinical case and analogous past cases, or on actions suggested by professional instinct. Hence, we may assume that a physician working in an emergency department often finds him/herself in the posi- tion of not being able to follow the procedure that should be optimal. He/she will refer to a less reliable procedure, because it is the only one compatible with the work setting.

\section{Opinion Report}

\section{Two systems}

Assessments based on immediate impressions are usually conceived as deceptive. In fact, many research data support the notion that, without reflection, our judgments are influenced by superficial aspects and are sometimes reinforced by social stereotypes: ${ }^{1}$ this leads to wrong choices.

Let us consider the two following sentences:

A) Cancer causes every year on average 24.14 deaths per 100 ;

B) Cancer causes every year on average 1286 deaths per 10,000

In an experimental setting, $75 \%$ of the subjects estimated that cancer gets worse in the second case, although actually in the first sentence the risk is $24.14 \%$ and in the second is $12.86 \%{ }^{2}$ The first value is almost twice the second: however, 1286 cases of cancer are more impressive than 24.14. The focus of the subject is captured by the entity in the average number of dead people and he/she neglects the size of the reference sample (100 in the first case and 10,000 in the second).

Another experimental task highlights how choices are influenced by the format used to present numeric data. In a study by Slovic, Monahan and MacGregor ${ }^{2}$ - see a detailed analysis of the task $\mathrm{k}^{3}$ - a sample of psychologists and psychiatrists were provided with information regarding the likelihood that a patient (Mr. Jones) would commit violent acts after being discharged from the clinic where he was hospitalized. Later, they were asked to express an opinion on the degree of perceived risk and to decide on the possible discharge of the patient. Two alternative versions were proposed, using different formats to present the data: in one case the risk was expressed as a probability (It is estimated that patients similar to Mr. Jones have a $20 \%$ chance of committing a violent act in months after discharge), in the other case relative frequencies were reported (It is estimated that, among patients similar to Mr. Jones, 20 of 100 commit a violent act in months following discharge). Analyzing the answers provided by a sample of experts, a significant effect of the presentation format on the evaluations emerged. When the data were expressed as relative frequencies (frequentistic format), the risk associated with the probability that the patient commits violent acts was perceived higher than when they were pre-
Correspondence: Alessandro Antonietti, Department of Psychology, Catholic University of the Sacred Heart, Largo Gemelli 1, 20123 Milan, Italy.

Tel. +39.02.72342909 - Fax: +39.02.72342280

E-mail: alessandro.antonietti@unicatt.it

Key words: metacognition, heuristics, medical decisions.

Received for publication: 3 December 2013.

Revision received: 5 May 2014.

Accepted for publication: 7 May 2014.

This work is licensed under a Creative Commons Attribution 3.0 License (by-nc 3.0).

(C) Copyright A. Antonietti et al., 2014

Licensee PAGEPress, Italy

Emergency Care Journal 2014; 10:2182

doi:10.4081/ecj.2014.2182

sented as probability (probabilistic format). Consequently, in the first case the respondents were more cautious in expressing their opinion in favor of the discharge of the patient with respect to the second case, when judgments were more favorable. The frequentistic format impresses more, and decisions are affected accordingly.

Errors in judgment and decision making, as those reported above, result from the fact that people rely on the impressions produced by the data. These errors could be avoided if individuals would take time to reflect on the data and to analyze them carefully. To do this, it is fundamental to resist the temptation to judge the situation on the basis of the immediate impressions, which corresponds to a common way of thinking. A cognitive effort should be targeted to move on to a different form of reasoning.

The idea that human beings can employ two different modes of thought is recurrent in psychology. ${ }^{4}$ The first system - called experiential $^{2}$ tacit $^{5}$ or System $1^{6}$ - is intuitive, since it is fast and action-oriented. It is activated in a non-intentional way and relies beyond the control of the individual, since the procedures on which it relies are pre-determined and implemented automatically. It is holistic because it allows the individual to take simultaneously into account more than one item at a time. It is based on mental associations and it is perceived as effortless. According to Epstein, ${ }^{7}$ this system is based on impressions.

The second system - called analytical, ${ }^{2}$ deliberative, ${ }^{5}$ rational or System $2^{6}$ - requires consciousness; it is intentional and sequential; it is driven by logic and follows specific rules. It is focused and requires time and effort. It operates mainly on the basis of abstract representations and it is relatively flexible. This system is not based on emotion- 
al cues and is not affected by them. The analytical processes can be decomposed into subprocesses that can access the level of consciousness serially (one at a time) and are accompanied by the feeling of effort. People rely deliberately on System 2 when they run into a situation that requires to be thoughtfully considered and they can often report the way they operate.

A dual vision of thinking processes is now widely accepted. ${ }^{8}$ The same perspective can be recognised in the distinction between the two modes of cognitive functioning identified by Kahneman. ${ }^{9,10}$ According to this author, in the intuitive mode (System 1) judgments and decisions are formulated automatically and quickly on the basis of impressions, which are emotionally characterised. The deliberative mode (System 2), on the other hand, is controlled and slow. According to Kahneman, System 1 originates errors. In fact, it is based on a process of attribute substitution: in some cases the individual assesses an objective attribute replacing it with the attribute which comes to mind most readily or attracts attention. This happens because the objective attribute is not accessible or requires a certain processing to be understood. The attribute that is replaced appears to be relevant and so overcomes the control, which System 2 should exert. An error of judgment implies the failure of both the System 1, which generated the error by replacing the attribute, and System 2 , which was not able to find out and correct it.

If we take the first case of error reported earlier (the one concerning the two descriptions of the average number of deaths caused by cancer), the objective attribute that should be considered is the relationship between the number of deaths and the size of the reference sample. This attribute, however, requires some mental processing. So the attribute number of deaths attracts the attention of the evaluator and is replaced, because of its salience and ease of processing, to the objective one. The replacement is not detected by System 2, and originates an erroneous judgment.

\section{Impressions are not misleading}

This vision, which leads to attribute to System 1 the responsibility for the malfunction of thinking processes and to System 2 (when it is correctly activated) the merit for its success, raises some concerns. First, System 1 does not always deceive. ${ }^{11}$ Some observations collected by Gladwell ${ }^{12}$ support the effectiveness of intuitive processing based on impressions. Patients know how to assess the skill of a doctor just by listening to his/her voice. The assessment of the personality of college students is more accurate when relies on a brief look at their room than when relies on tests. Marriage counselors are able to provide, watching only a few fragments of videotaped husband-wife interaction sessions, which couple will ask for a separation and which will stay together. In addition, it has been proved that patients are able to catch simple but reliable communication signals and use them in order to decide whether they should trust their doctors. ${ }^{13}$

The advantages of intuition have been documented by Dijkserhuis in a series of experiments where, for example, some car models were described to participants, providing them 4 or 12 characteristics for each model. The task was to identify the best model. In one condition the subjects had 4 min to carefully consider the characteristics, in the other condition the subjects had the same time, but they were also engaged in a different task, so that they could not reflect on the characteristics of the cars (condition of decision without deliberation). The best choices were made by the first group when 4 characteristics were available and in the second group when 12 characteristics were available. So, when time is not enough to analytically consider all available information, the best strategy is to rely on immediate impressions. ${ }^{14}$

Why can System 1 be effective? The impressions it produces allow understanding the essential aspects of a situation: this helps individuals to extrapolate quickly the most important elements. The action of simplification accomplished by intuition aims at linking elements to each other to provide a preliminary overview of the situation. In short, intuition offers a preliminary selective representation (where the affordances - namely, the functionally salient features - and the structural aspects of the situation are highlighted) and holistic (so as to lead people to grasp the patterns of relationships that give a complete idea of the situation). Hence, System 1 does not necessarily deceive, but can also help people.

On the other hand, System 2 has some limitations of its own. First of all, analytical processes may not be optimal because of the excessive cognitive load that they imply. Analytical thinking is efficient in stable conditions, when enough time is available and when the criteria that have to be applied are clear and unambiguous. In particular, it is important that System 2 can access the specific resources needed to monitor and control the flow of thought. Its activation may require too much time, and this may not be viable in everyday circumstances. In addition, its optimal functioning needs conditions that cannot be managed by the human mind.

\section{The heuristics}

According to Simon, ${ }^{15}$ thinking processes are affected by the constraints of the cognitive abilities of human beings. A first limitation concerns the process of acquiring information from the environment. Such a process, in fact, is necessarily selective, since the cognitive system is not able to consciously register the immense amount of stimuli from the external world. The limits also concern both the time available to pay attention and the amount of information on which it is possible to focus simultaneously. The memory system shows constraints too: short-term memory has a limited capacity in terms of time and amount of information retained and long-term memory has limitations related to the possibility of information retrieval. As a direct consequence, people develop heuristic strategies (or, more simply, heuristics) to evaluate, judge and make a decision consistently with the complexity of the task and the constraints of the cognitive system. Most of the situations that we face in everyday life require speed and the ability of making choices based on little information. ${ }^{16}$ Heuristics operate in contexts with some impact on social life such as, for example, choosing whom to entrust with the managing of savings, or selecting an insurance, rather than undergoing diagnostic tests. ${ }^{17}$ In all these situations people, whether they are experts in that field or not, rely on rules of thumb ${ }^{18}$ that simplify the problem.

A particular category of heuristics are the fast-and-frugal heuristics, which allow people to browse available information very quickly and stop the search process when they find a satisfactory solution..$^{19,20}$ As an example, we may consider a situation where people have to buy a drug-counter to solve problems such as sore throat, cough, or allergies. How do individuals choose these drugs? In a study ${ }^{21}$ it was shown that participants considered less than half of available information. Respondents adopted a very frugal approach in the acquisition of information showing a clear preference to consider a relatively small set of notions in order to decide which drug should be purchased. Which rules were more precisely applied in the exploration of available information? Two distinct approaches can be identified: the feature-wise approach and the globalwise approach. The feature-wise approach leads people to polarize the attention on a single characteristic at a time and to continuously compare this feature on the various products (for example, focusing on side effects and evaluating all drugs with reference to this characteristic). The global-wise approach, by contrast, leads individuals to explore only the features that belong to a single drug at a time and never to make comparisons. Data revealed that about $75 \%$ of the participants followed an approach oriented to consider the characteristics belonging to a drug at a time and systematically considered a limited number of characteristics of the drug. This frugal approach resulted functional in $78 \%$ of cases. 


\section{Heuristics in medical decisions}

Heuristics can be applied in health care not only by patients, but also by medical professionals. ${ }^{22}$ It was observed, for example ${ }^{23}$ that the emergency arisen in the emergency department of the city of New York as a result of the terrorist attack on the Twin Towers was adequately tackled thanks to the system Simple Triage And Rapid Treatment (START), which allows a professional to assess the general health of the injured person in $60 \mathrm{sec}$. The system is based on the recognition of the presence/absence of only three parameters (respiration, blood circulation, mental state) under which it is possible to figure out which units of the second level of intervention is appropriate to direct the patient.

If procedures are too complicated, healthcare professionals do not apply them. Smith and Gilhooly ${ }^{24}$ realised that the guidelines of the Scottish Office Health Department for psychiatric diagnosis - according to which a drug has to be prescribed to the patient if he/she shows at least one of two key symptoms and at least four out of seven other symptoms are present for at least two weeks - were actually disregarded in medical practice because they were too complicated. The authors presented to physicians 20 cases, providing for each of them eight types of information (gender, duration of depressive symptoms, presence of suicidal thoughts, feelings of uselessness and/or guilt, disturbed sleeping, decreased appetite and/or weight, difficulty in concentrating, attitude to care). The decision to prescribe an antidepressant was taken on the basis of limited information: $58 \%$ of doctors used two pieces of information, $25 \%$ used one piece of information, 9\% used three pieces of information and only $8 \%$ used more than three pieces of information.

Dhami and Harries ${ }^{25}$ described 100 patients to physicians, providing for each of them twelve types of information (cholesterol, blood pressure, age, gender, atherosclerosis, alcohol consumption, smoking, diabetes, diet, weight, attitude toward care, ischemia). Then they asked the doctors if they would or would not prescribe a drug in order to decrease lipids. The doctors used only one or two pieces of information, behaving as if they had applied these three simple rules:

Search rule $=$ Consider information in order of importance;

Adjust stop=Stop searching as soon as the first useful datum permits you to decide;

Decision rule=Prescribe or not the medicine according to the piece of information mentioned above

For example, if a physician believes that the three most important notions are cholesterol, age and ischemia (search rule), he/she, in evaluating the patient, considers first the cholesterol level and if this is not particularly high, he/she proceeds to the second type of information, i.e., age. If this is not high, he/she moves to the third kind of information: did the patient report cases of ischemia in his/her family? If so, the doctor proceeds over (stopping rule) and prescribes the drug (decision rule), otherwise he/she does not prescribe the drug.

The role which the heuristics have in medical decision making has recently been illustrated by Cervellin, Borghi and Lippi. ${ }^{26}$ They claimed that clinical judgment can be considered as a synthesis of intuition (mainly based on Gestalt principles) and analytical approach (grounded on Bayesians principles). In agreement with Kienle and Kiene, ${ }^{27}$ they observed that the model of technical rationality taught to medicals has been proven to be grossly oversimplified and only applicable to simple and repetitive situations, but not to the complexity that generally characterises professional situations. Authors showed the role of Gestalt principles in helping clinicians in everyday work tasks like physical examinations. Medical students are usually trained to read the ECG as a sequence of cues (frequency, rhythm, conduction, and so on). This is very helpful for novice because they risk to jump the diagnosis at the price of missing important features. However, Gestalt perception is dominating in skilled internists and cardiologists, who base the diagnosis on the overall picture and only later they comment single aspects of the tracing. In the mind of the expert physician, a sort of automated, fast, flowchart may suddenly guide his/her Gestaltic thought.

Are medical decisions based on fast-and-frugal heuristics, such as those described above, valid? It would seem so. ${ }^{28}$ Fisher and colleagues $^{29}$ analysed data collected over two years and referring to 253 children diagnosed with pneumonia; 32 of these have revealed, on the basis of serological testing and nasopharyngeal secretions, Mycoplasma pneumonia. In these cases it is important to prescribe immediately the appropriate antibiotic: this requires physicians to be able to distinguish quickly pneumonia Mycoplasma from pneumonia Chlamydophila. The authors pointed out that a diagnosis based solely on the duration of fever and age of the patient was as accurate as the one based on the outcomes of the clinical examination, which obviously takes more time.

Similarly, Kattah and colleagues ${ }^{30}$ examined $^{2}$ the medical records of 121 patients with acute vestibular syndrome, a complex of symptoms that may underpin vestibulopatia (such as labyrinthitis) or stroke. It is obvious that in these cases it is important to move quickly from the symptoms to a diagnosis. An examination by magnetic resonance allows doctors to decide which of the two diagnoses has to be given, but it takes time (and it is expensive). A neurological examination is quicker and cheaper. Even more fast-and-frugal is a diagnosis based on the three indicators (vestibuloocular reflex, nystagmus, testing the vertical misalignment of the eyes), which requires a minute and achieves accuracy levels comparable to those of the other methods.

Another example in the psychiatric domain has been provided by Jenny and colleagues, ${ }^{31}$ who showed that the detection of depressed mood based on a fast-and-frugal decision tree involving only four issues (crying more often than usual, hating oneself, being discouraged, feeling to have failed in one's life) is superior to more complex diagnostic systems.

\section{Metacognitive skills}

To summarise, when we have to judge and take decisions, we can rely on three different ways of thinking, each with its own strengths and weaknesses. First, our intuitive system can immediately suggest some answers. This system proceeds basically outside of our awareness, in the sense that we do not have information on how things are going on in our minds, but we are aware only of their final outcome. Then, we have the heuristic strategies, which offer frugal procedures to simplify situations and find satisfactory solutions. Heuristics are placed at an intermediate level of awareness. Their application sometimes happens automatically, but we can reconstruct the principles on which they are based. Furthermore, these strategies, in contrast to what happens with intuitions, can be applied and taught intentionally. Finally, there are thinking processes based on logical criteria and normative principles. They allow us to reflect on the situation coldly and analytically, to weight pros and cons and to identify possible sources of error.

In some cases, researchers describe these mental systems as mutually exclusive, or at least as competing with one another. Yet, the flexible use of various systems seems to be the optimal situation. In this case, the individual activates the system that is more relevant to the given situation and eventually passes to another system when he/she realizes that the previous one is inadequate. In the field of emergency care it is important that professionals are able to discern quickly when impressions are reliable or when carrying out a detailed examination of the situation is preferable. But how can a physician manage the interaction among the different systems in a flexible way so to identify the mental system that is more relevant to a specific case?

It seems that metacognitive competence is required. Such a competence consists in the ability to self-regulate cognitive processes so as to match the needs of the moment. To do so, individuals have to pay attention to their cog- 
nitive processes and understand how they can be trusted and what is the best way to handle them. More specifically, metacognitive competence includes different sub-skills: i) having adequate beliefs about the way the mind works; ii) being aware of the way your own mind works; iii) being able to control the operation of your own mind based on information you get from the knowledge of the way you think and based on the beliefs that you have about the general functioning of the mind.

First of all, it is important that the beliefs about cognitive processes correspond to the way these processes actually occur. For example, if I am convinced that reflecting too much on clinical cases is always counterproductive, I will seldom use analytical thinking. If I believe that inattention causes errors in diagnosis, when I have to take care of a patient in the emergency department (ER) I will do my best to stay focused. Secondly, I need to be aware of my personal characteristics and of how I proceeded in the past and of how I am currently carrying out my reasoning. If I know that I am an impulsive person, who tends to be affected by temporary irritation, and I recognize that this attitude has caused me to commit serious mistakes in the past, I will try to control this tendency by examining whether in the situation that I am facing I am running the risk of committing similar mistakes.

However, adequate knowledge and beliefs are not sufficient. Bransford, Sherwood and Sturdevant ${ }^{32}$ pointed out that, sometimes, cognitive difficulties arise not because we do not have the proper knowledge about the strategies to be applied, but because we fail to make use of that knowledge. Why does this happen? Because we may lack awareness about the mental mechanisms that we activate ${ }^{33}$ and this reduces our capacity for self-regulation. This also appears in reference to the choices made by physicians. ${ }^{34}$ From a practical standpoint, higher metacognitive competence should be fostered when training medical staffs, so that professionals are able to identify the mode of thinking - intuitive, heuristic or analytical - that is best suited to make the choices required by the clinical cases that they are facing.

\section{Conclusions}

Which practical indication can we derive in order to enhance metacognitive competence in professionals working in the ER? From what we have been discussing in this paper, it is clear that ER professionals can rely on different systems, which are related to different thinking modalities, characterised by different strengths and weaknesses. Metacognition is the key skill that can lead these professionals to better manage their thinking processes according to the type of emergency in a specific setting.

Hence, a main practical implication, which logically derives from our discussion, is that, as a first step, specific metacognitive trainings should be implemented. These trainings should consist first of hints aimed at prompting individuals to reconstruct retrospectively the way they usually manage clinical situations and to become aware of the concurrent thoughts and emotions. The second step is inducing trainees to assess their prevalent mode of thinking by inviting them to check out how often they apply each of the three cognitive systems mentioned previously (intuitive, heuristic and analytical). In the following phase professionals should test if and why the preferred thinking style is functional in the work setting where they operate. This kind of reflections usually leads trainees to detect the specific circumstances in which each system is suited to achieve the desired goals. The final step includes supervised activities in which the physicians are taught to identify personal cues that can help them to activate the proper thinking procedure according to specific cases. This type of training program on the one hand results in providing doctors with a series of concrete hints to be applied in the actual practice and, on the other hand, in fostering a reflective attitude which enables them to monitor and revise continuously their behavior, so to adapt it to the working conditions that can change as a consequence of technical innovations, organizational transformations and increase in professional expertise as well.

\section{References}

1. Baldi PL, Iannello P, Riva S, Antonietti A. Socially biased decisions are associated to individual differences in cognitive reflection. Stud Psychol 2014;55:265-71.

2. Slovic P, Finucane M, Peters E, MacGregor DG. The affect heuristic. In: Gilovich T, Griffin D, Kahneman D, eds. Heuristics and biases. New York, NY: Cambridge University Press; 2002. pp 397-420.

3. Iannello P, Antonietti A. Format bias: is it really matter of frequency vs. probability? In: Tateo L, Iannaccone A, Storti G, eds. Decisa-mente. Teorie, processi e contesti di decision making. Rome: Aracne Editrice; 2011. pp 101-8.

4. Evans JSBT. Thinking twice. Two minds in one brain. Oxford: Oxford University Press; 2010.

5. Hogarth RM. Educating intuition. Chicago: University of Chicago Press; 2001.

6. Stanovich KE, West RF. Individual differences in reasoning: implications for the rationality debate? Behav Brain Sci 2002;23:645-726.

7. Epstein S. Integration of the cognitive and the psychodynamic unconscious. Am Psychol 1994;49:709-24.

8. Macchi C, Over D, Viale R. Dual process theories of human thought: the debate. Mind \& Society 2012;11:1-2.

9. Kahneman D. Maps of bounded rationality: a perspective on intuitive judgment and choice. Wiksell International/The Nobel Foundation; 2002.

10. Kahneman D. Thinking. Fast and slow. New York, NY: MacMillan; 2011.

11. Dane E, Pratt MG. Exploring intuition and its role in managerial decision making. Acad Manage Rev 2007;32:33-54.

12. Gladwell M. Blick. The power of thinking without thinking. New York, NY: Back Bay Books; 2005.

13. Riva S, Monti M, Iannello P, et al. A preliminary mixed-method investigation of trust and hidden signals in medical consultations. PlosONE 2014;9:e90941.

14. Dijksterhuis AP, Bos MW, Nordgren LF, Van Baaren RB. On making the right choice: the deliberation-without-attention effect. Science 2006;311:1005-7.

15. Simon HA. Rational choice and the structure of the environment. Psychol Rev 1956;63:129-38.

16. Gigerenzer G, Brighton H. Homo heuristicus: why biased minds make better inferences. Top Cogn Sci 2009;1:107-43.

17. Gigerenzer G, Selten R. Bounded rationality: the adaptive toolbox. Cambridge, MA: MIT Press Cambridge; 2001.

18. Gigerenzer G. Gut feelings. The intelligence of the unconscious. New York, NY: Viking; 2007.

19. Gigerenzer G. Adaptive thinking: rationality in the real world. Oxford: 0xford University Press; 2000.

20. Gigerenzer G, Todd PM, ABC Research Group. Simple heuristics that make us smart. New York-0xford: 0xford University Press; 1999.

21. Riva S, Monti M, Antonietti A. Simple heuristics in over the counter drugs' choices. A new hint for medical education and practice. Adv Med Educ Pract 2011;2:59-70.

22. Riva S, Monti M, Iannello P, Antonietti A. The representation of risk in routine medical experience: what actions for the contemporary health policy? PlosONE 2011;7:e48297.

23. Cook L. The World Trade Center attack. The paramedic response: an insider's view. Crit Care 2001;5:301-3.

24. Smith L, Gilhooly K. Regression versus fast and frugal models of decision-making. The case of prescribing for depression. Appl Cognitive Psych 2000;20:265-74. 
25. Dhami MK, Harries C. Fast and frugal versus regression models of human judgement. Think Reasoning 2001;7:5-27.

26. Cervellin G, Borghi L, Lippi G. Do clinicians decide relying primarily on Bayesians principles or on Gestalt perception? Some pearls and pitfalls of Gestalt perception in medicine. Intern Emerg Med 2014;9:513-9.

27. Kienle GS, Kiene H. Clinical judgement and the medical profession. J Eval Clin Pract 2010;17:621-7.

28. Marewski JN, Gigerenzer G. Heuristic decision making in medicine. Dialogues Clin Neurosci 2012;14:77-89.
29. Fisher JE, Steiner F, Zucol F, et al. Use of simple heuristics to target macrolide prescription in children with communityacquired pneumonia. Arch Pediat Adol Med 2002;156:1005-8.

30. Kattah JC, Talkad AV, Wang DZ, et al. HINTS to diagnose stroke in acute vestibular syndrome. Stroke 2009;40:3504-10.

31. Jenny MA, Pachur T, Lloyd Williams S, et al. Simple rules for detecting depression. J Appl Res Mem Cogn 2013;2:149-57.

32. Bransford JD, Sherwood RD, Sturdevant T. Teaching thinking and problem solving. In: Baron JB, Sternberg RJ, eds. Teaching thinking skills: theory and practice. New
York, NY: WH Freeman Publ.; 1987. pp 16281.

33. Colombo B, Iannello $\mathrm{P}$, Antonietti A. Metacognitive knowledge of decision-making: an explorative study. In: Efklides A, Misailidi P, eds. Trends and prospects in metacognitive research. New York, NY: Springer; 2010. pp 445-72.

34. Donati A, Iannello P, Perucca V, Antonietti A. [Come decidono i medici nei servizi di Pronto Soccorso? Uno studio sulla consapevolezza metacognitiva]. [Article in Italian]. Emerg Care J 2012;8:18-25. 